\title{
Solid acids and their use as environmentally friendly catalysts in organic synthesis*
}

\author{
Karen Wilson and James H. Clark \\ Green Chemistry Group, Department of Chemistry, University of York, Heslington, \\ York YO10 5DD, UK
}

\begin{abstract}
Tightening environmental legislation is driving the fine and speciality chemicals industries to consider alternative processes that avoid the use of conventional mineral acids. The use of heterogeneous catalysts in these processes would vastly simplify catalyst removal, minimizing the amount of waste formed. However, diffusion limitation of liquids within porous solids dictates that effective solid acids for liquid-phase reactions require the use of mesoporous materials $<20-100 \AA$. Recent developments in materials chemistry has led to the discovery of a family of ordered mesoporous silicas which opens up new possibilities for preparing solid-acid catalysts for liquid-phase reactions. This review concentrates on recent developments in the synthesis of new mesoporous solid acids for liquid-phase organic synthesis.
\end{abstract}

\section{INTRODUCTION}

Catalytic technologies play a key role in the economic development and growth of the chemicals industry and contribute to around 20\% of world GNP. A major emerging and challenging area of heterogeneous catalysis is that of environmental pollution control, with tightening legislation on the release of waste and toxic emissions having serious implications for the chemical industry. While heterogeneously catalyzed processes are widely used within the petrochemical industry, many fine and specialty chemicals manufacturers rely on homogeneously catalyzed liquid-phase reactions. Many of these processes were developed at the turn of the century, and focused on product yield, disregarding the environmental impact of inorganic waste and toxic by-products formed during the reaction [1].

A wide range of liquid-phase industrial reactions rely on the use of inorganic or minerals acids. While many of these processes are catalytic, some require (e.g., acylation using $\mathrm{AlCl}_{3}$ ) stoichiometric amounts of acid. Final isolation of the product necessitates aqueous quenching and neutralization steps to remove the acid, resulting in enormous quantities of hazardous waste, with the cost of disposal of this waste often outweighing the value of the product. Tightening legislation on the emission of hazardous pollutants is driving the industry toward the implementation of innovative "clean technology" including the use of alternative heterogeneously catalyzed processes [2]. Heterogeneous catalysts offer several intrinsic advantages over their homogeneous counterparts: ease of product separation and catalyst reuse; bifunctional phenomena involving reactant activation/spillover between support and active phases; and process advantages through reactor operation in continuous flow versus batch configuration. However, to maintain economic viability, a suitable heterogeneous system must not only minimize the production of waste, but should also exhibit activities and selectivities comparable or superior to the existing homogeneous route.

Zeolites are widely used within the petrochemical industry in acid catalyzed processes, and there are several reviews concerning recent developments in their use in the synthesis of fine and specialty chemicals $[3,4]$. When performing reactions in the liquid phase, it is important that product diffusion from the active site is rapid to minimize catalyst deactivation. Unfortunately, the use of zeolites under

*Pure Appl. Chem. Vol. 72, No. 7, 2000. A special topic issue on green chemistry. 
these conditions is restricted by their small pore sizes of around $<8 \AA$, which makes them unsuitable for reactions involving bulky substrates. However, recent developments in materials chemistry has led to the discovery of the M41S family of mesoporous molecular sieves [5] offering pore sizes in the range 20-100Å which opens up new possibilities for liquid-phase acid catalysis. This article will concentrate on the developments in liquid-phase catalysis using solid-acid catalysts based on mesoporous oxide materials.

\section{Solid acids in organic synthesis}

\section{Properties of solid acids}

Solid-acid catalysts are generally categorized by their Brønsted and/or Lewis acidity, the strength and number of these sites, and the morphology of the support (e.g., suface area, pore size). The synthesis of pure Brønsted and pure Lewis acid catalysts attracts a great degree of academic interest, although the latter is harder to achieve because Brønsted acidity often arises from Lewis acid-base complexation, as illustrated in Scheme 1.

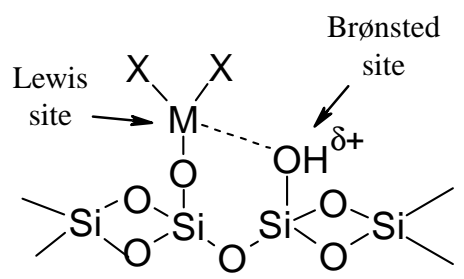

Scheme 1 Brønsted acidity arising from inductive effect of Lewis acid center coordinated to a silica support.

To obtain high selectivity toward the desired products in a synthetic reaction all these properties must be considered. For example, acetal formation and hydrolysis reactions generally require medium acid strength sites, while electrophilic additions of alcohols or water to olefins, skeletal rearrangements, esterification, and alkylation reactions require strong acid sites. Likewise, the importance of the nature of the acid site is demonstrated in Friedel-Crafts alkylation reactions, where Lewis acid sites are required for alkylation of toluene using benzyl chloride, while Brønsted sites are preferred for reactions using benzyl alcohol [6]. In addition, steric constraints imposed by the pore structure of the solid acid can influence the reaction pathway resulting in "shape-selective catalysis". Recent development in the preparation of mesoporous materials has allowed liquid-phase reactions with bulky substrates to be performed.

\section{Acid catalysis using mesoporous materials}

M41S mesoporous molecular sieves [5] are prepared using an anionic templating mechanism in which growth of the silica framework occurs around a quaternary ammonium salt micelle. By varying the chain length of the alkyl chain of the quaternary salt an ordered pore structure of predefined size is observed on template removal. MCM-41 can be synthesized in either a pure silica form, or with both tetrahedrally coordinated silicon and aluminum atoms incorporated into the framework. The latter results in the formation of Brønsted acid sites via the generation of bridging SiOHAl hydroxyl groups, similar to those found in amorphous alumino-silicates [7]. The large regular pore structure of MCM-41 materials make them suitable for liquid-phase acid catalysis by enabling rapid diffusion of reactants and products through the pores, thus minimizing consecutive reactions. 
Liquid-phase Friedel-Crafts alkylation and acylation reactions [8-12] have been reported using aluminosilicate MCM-41. The advantages of increased pore size are clearly demonstrated in the alkylation of 2,4-di-tert-butylphenol using cinnamyl alcohol [8]. MCM-41 gives a high yield of the desired product dihydrobenzopyran, whereas with US-Y zeolites problems of diffusion limitation are apparent with only trace amounts of this product being formed. The large pore structure of MCM-41 also enables the alkylation of anthracene and napthalene by tert-butene to be performed [10].

MCM-41 has also been used for the acylation of 2-methoxynapthalene with acetic anhydride [11]. Acylation at the 6 position produces an intermediate of importance for the production of the antiinflamatory drug Naproxen. However, while MCM-41 exhibits high activity in this reaction with $100 \%$ conversion of 2-methoxynapthalene being observed at $100{ }^{\circ} \mathrm{C}$, the selectivity towards the 6 position acylated product is low $(<10 \%)$. Other reactions catalyzed using MCM-41 include the acetalysation of bulky aldehydes [13], glucosidation [14] and aldol condensations [3].

A variation of the MCM family of materials was reported by Pinnavaia et al [15] who developed a neutral templating method using long-chain alkylamines to form hexagonal mesoporous molecular sieves (HMS materials). This preparative route has the advantage that template removal is simpler and more environmentally benign, requiring only solvent extraction instead of calcination or ion exchange. The use of a neutral templating process also opens possibilities for incorporation of organo-functionalized silanes into the silica framework during the sol-gel process. Organo-modified silicas are widely used in chromatographic and other analytical applications [16], and the techniques for their preparation are well documented. For example, silica functionalized with sulfonic acid groups is frequently used for binding metal ions [17]. The chemistry used to prepared these materials has recently been exploited to prepare a pure sulfonic acid-functionalized mesoporous silica [18,19], which is a solid Brønsted acid.

These sulfonic acid-functionalized mesoporous silicas are prepared using $\mathrm{H}_{2} \mathrm{O}_{2}$ to oxidize thiol functionalized silicas previously synthesized via a sol gel or post-modification grafting route. Sol gel preparation of thiol functionalized silica was performed by co-condensation of $\mathrm{Si}(\mathrm{OEt})_{4}$ and 3-mercaptopropyltrimethoxysilane (MPTS) using either neutral or ionic templating methods, with subsequent oxidation producing $\mathrm{HMS}-\mathrm{SO}_{3} \mathrm{H}$, and $\mathrm{MCM}-\mathrm{SO}_{3} \mathrm{H}$, respectively [18]. These sulfonic acids exhibit high catalytic activity in the preparation of bisfurylalkanes from 2-methylfuran and acetone. Higher conversions of 2-methylfuran and selectivities toward 2,2-bis(5-methylfuryl)propane were observed compared to reactions using zeolites H- $\beta$ or H-US-Y which rapidly deactivate due to oligomerization of 2-methylfuran. Both sol gel and post-modified sulfonic acid silicas also exhibit considerable selectivity toward monoglyceride formation during the esterification of polyols with fatty acids [19].

The concept of using sulfonic acids as Brønsted acid catalysts is not new, with commercial sulfonated resins Amberlyst-15 and Nafion-H (based on sulfonated polystyrene and perfluorinated sulfonic acid resins, respectively) being widely available. The main advantage of silica-supported solid sulfonic acid over these systems is its higher surface area of $400-900 \mathrm{~m}^{2} \mathrm{~g}^{-1}$ compared to $<1 \mathrm{~m}^{2} \mathrm{~g}^{-1}$ for resins. Despite its low surface area, Nafion- $\mathrm{H}$ has been used in a wide range of organic reactions [20] including alkylation, acylation, nitration, etherification, and esterification reactions. Recently, in an attempt to overcome the low surface area of these ion-exchange resins, a group of workers has developed Nafion-silica composites, in which Nafion particles are incorporated into the silica structure during the sol gel process [21-23]. These composite materials exhibit high activity in alkylation, esterification, and more recently have been used in Fries rearrangement [24]. A variation of these materials was also reported, which involved the functionalization of silica with perfluoro sulfonic acid [25] which was synthesized from $(\mathrm{OEt})_{3} \mathrm{Si}\left(\mathrm{CH}_{2}\right)_{3}\left(\mathrm{CF}_{2}\right) \mathrm{O}(\mathrm{CF})_{2} \mathrm{SO}_{2} \mathrm{~F}$ and tetramethoxysilane. However, the complexity of the preparation of $(\mathrm{OEt})_{3} \mathrm{Si}\left(\mathrm{CH}_{2}\right)_{3}\left(\mathrm{CF}_{2}\right) \mathrm{O}(\mathrm{CF})_{2} \mathrm{SO}_{2} \mathrm{~F}$ means that this material is not really cost effective when compared to the use of MPTS discussed above.

\section{Post-modified mesoporous solid acids}

Not only does the incorporation of framework heteroatoms into mesoporous materials allow them to be used as solid acids, but the high surface areas of both amorphous and ordered materials means they are

(C) 2000 IUPAC, Pure and Applied Chemistry 72, 1313-1319 
ideal supports for catalytically active phases such as heteropoly acids, metal oxides, alkoxides, or halides. There are a range of supported reagents based on either metal salts such as $\mathrm{ZnBr}_{2}$ [26] or $\mathrm{ZnCl}_{2}$ [27], physisorbed onto silica, or cation-exchanged montmorillonites [28]. For example, $\mathrm{ZnCl}_{2}$ supported on $\mathrm{K} 10$ is an extremely efficient catalyst for benzylation reactions and other alkylations involving alkyl halides. More recently, a new Lewis acid catalyst prepared by supporting $\mathrm{Zn}\left(\mathrm{CF}_{3} \mathrm{SO}_{2}\right)_{2}$ on silica has been reported, which exhibits extremely high activity and turnover numbers in the rearrangement of $\alpha$ pinene oxide [29]. Diels Alder reactions have also been reported over MCM-41 [30] and organolanthanidefunctionalized MCM-41 [31]. However, while at present, these "supported reagents" offer one of the few ways of forming a pure heterogeneous Lewis acid catalyst, they are limited to use in reactions in nonpolar media due to weak interactions between the metal salt and the support.

Post modification that involves direct reaction of hydroxyl groups on an oxide support with a Lewis acid metal salt produces a much more stable solid acid. However, using these methods, both Lewis and Brønsted acid sites are formed due to polarization of surface hydroxyl groups by the Lewis center as previously explained in Scheme 1. Such grafting methods have been used successfully for preparing a heterogeneous form of $\mathrm{AlCl}_{3}$ which exhibits high activity in Friedel-Crafts alkylation reactions [32]. By refluxing $\mathrm{AlCl}_{3}$ with $\mathrm{SiO}_{2}$ in a nonpolar solvent, reaction according to Scheme 2 is believed to occur. Interaction of $\mathrm{AlCl}_{\mathrm{x}}$ with adjacent hydroxyl groups also results in the formation of Brønsted acid sites. When $\mathrm{AlCl}_{3}$ supported on amorphous $\mathrm{SiO}_{2}(70 \AA)$ was used in the alkylation of benzene with alkenes, in addition to the added advantage that the heterogeneous catalyst can be recycled, greater selectivity towards monalkylated products were observed compared to homogeneous $\mathrm{AlCl}_{3}$. More recently, $\mathrm{AlCl}_{3}$ supported on crystalline HMS materials with an average pore size of $30 \AA$, have been shown to exhibit even higher selectivities towards monoalkylated products [33,34].

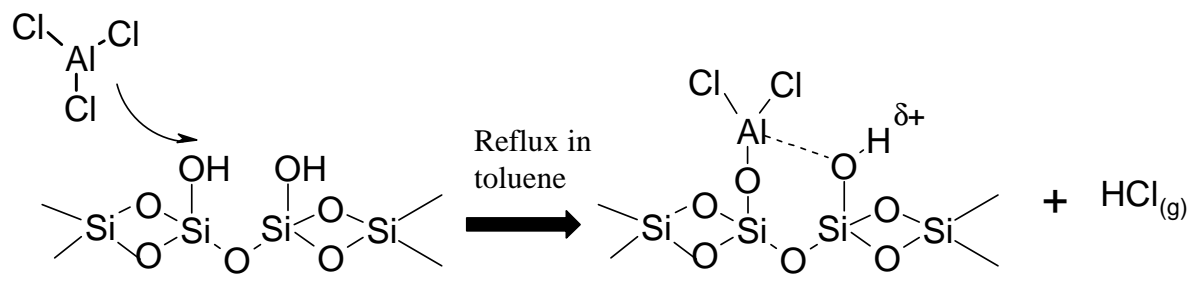

Scheme 2 Reaction of $\mathrm{AlCl}_{3}$ with $\mathrm{SiO}_{2}$ to form a highly active alkylation catalyst.

Similar preparative methods should be applicable to the heterogenization of other Lewis acids, however there are few reports of other examples in the literature apart from supported $\mathrm{FeCl}_{3}$ [35]. The grafting technique used depends on the stability of the dehalogenated intermediate $\mathrm{O}-\mathrm{MX}_{2}$ or $\mathrm{O}_{2} \mathrm{MX}$. The development of supported $\mathrm{BF}_{3}$ solid-acid catalyst is an interesting example where using reflux conditions to graft the Lewis acid to the surface is detrimental to catalyst activity [36,37].

Previous attempts to support $\mathrm{BF}_{3}$ have concentrated on reacting gas-phase $\mathrm{BF}_{3}$ with oxide supports $[38,39]$. Recently, a novel method to prepare supported $\mathrm{BF}_{3}$ catalysts by impregnating inorganic oxide supports with liquid $\mathrm{BF}_{3}$ precursors was reported. This method takes advantage of the fact that in the liquid phase $\mathrm{BF}_{3}$ readily forms complexes with inorganic or organic molecules $\mathrm{HX}$ (e.g., $\mathrm{HX}=\mathrm{H}_{2} \mathrm{O}$, $\mathrm{EtOH}, \mathrm{Et}_{2} \mathrm{O}$ ), to form a protic complex $\left[\mathrm{BF}_{3} \mathrm{X}\right]^{-}[\mathrm{H}]^{+}$. When supported on an oxide support this allows the Brønsted acid strength of the catalyst to be tuned via the formation of $\left[\mathrm{Si}^{-} \mathrm{OBF}_{3}\right]^{-}\left[\mathrm{H}_{2} \mathrm{X}\right]^{+}$sites, where the resulting Brønsted acidity is believed to be related to the $\mathrm{pKa}$ of $\mathrm{H}_{2} \mathrm{X}^{+}$. Reactivity studies using phenol alkylation as a model reaction to test the relationship between proton-donating ability of supported $\mathrm{BF}_{3}$ complexes and catalytic activity reveal that catalysts possessing enhanced Brønsted acidity exhibit increased phenol conversions. The most active and air-stable version of this catalyst is prepared 
by supporting a $\mathrm{BF}_{3}\left(\mathrm{H}_{2} \mathrm{O}\right)_{2}$, precursor on $\mathrm{SiO}_{2}$ using ethanol as the solvent. This catalyst, while being highly active for phenol alkylation, is also capable of performing esterification, etherification, and Claisen Schmidt condensation reactions [40].

The final class of acid catalyst to be discussed here concerns Keggin-type heteropoly acids (HPAs), of general formula $\mathrm{H}_{(8-x)} \mathrm{XM}_{12} \mathrm{O}_{40}$ where $\mathrm{X}=\mathrm{Si}^{4+}, \mathrm{P}^{5+}$ and $\mathrm{M}=\mathrm{W}^{6+}$ or $\mathrm{Mo}^{6+}$. These materials exhibit very strong Brønsted acidity approaching the superacidic region [41], and there are many reviews discussing various aspects of catalysis using HPAs, the most recent of which summarizes all recent developments in this field [42]. Solid Keggin-type HPAs have low surface areas of $1-5 \mathrm{~m}^{2} \mathrm{~g}^{-1}$, so the availability of high surface area mesoporous materials has led researchers to investigate the use of HPAs immobilized on MCM- or HMS-based supports in liquid-phase organic reactions. In addition to the increased dispersion, supported HPAs also have an advantage that coke formation is inhibited during reactions [43]. This may be attributed to a reaction of the HPA with $\mathrm{SiO}_{2}$ to form a new type of Brønsted site with reduced acid strength according to $\mathrm{H}_{3} \mathrm{PW}_{12} \mathrm{O}_{40}+\mathrm{Si}-\mathrm{OH} \rightarrow \mathrm{Si}_{\mathrm{m}}^{+}\left(\mathrm{H}_{3-\mathrm{m}} \mathrm{PW}_{12} \mathrm{O}_{40}\right)^{\mathrm{m}-}+\mathrm{mH}_{2} \mathrm{O}$.

Supported HPAs can be prepared either by post-modification of the mesoporous support, or can be incorporated into the pore structure during the sol gel process [44]. These silica 'included' materials are more resistant towards leaching of the HPA component.

Silica-supported and -included heteropoly acids exhibit high activity in a range of acid-catalyzed reactions, including the alkylation of benzene by 1-octene [43], trans-de-tert-butylation, and the alkylation of phenols [45,46], in addition to a range of esterification [47] and hydrolysis [44] reactions. More recent studies on esterification reactions using heteropoly acids supported on MCM-41 [48] suggest that the formation of $\mathrm{H}_{2} \mathrm{O}$ during reaction can result in re-dispersion of HPA salt on the surface. Silicasupported PW also exhibit higher activities than their corresponding unsupported equivalents in Diels Alder reactions [49]. Again, this improved activity is believed to be due to reduced coking or site deactivation by heavy organic material.

The availability of controlled pore-size mesoporous materials has led to new and exciting research being performed in the development of solid-acid catalysts for liquid-phase reactions. Through interdisciplinary collaboration between chemists, materials scientists, and chemical engineers in both industry and academia there should be many exciting developments leading to viable alternatives to traditional homogeneously catalyzed processes.

\section{GREEN CHEMISTRY INITIATIVES INTHE UNITED KINGDOM}

There has been a significant growth in interest in the principles of green chemistry in the United Kingdom within the last two years. Clean technology and in particular "clean synthesis" programs were used by the UK Research Councils to encourage research into novel and environmentally benign chemical processes and products. A Clean Technology Fellowship scheme administered by the Royal Academy of Engineering further stimulated these activities and helped to establish centers of excellence. There are now internationally recognized large research groups working on clean chemical technology in several leading UK universities including Queens Belfast, Leeds, Nottingham, and York. The enhanced level of green chemistry research and other factors has also helped to stimulate industrial interest, and there are now a growing number of good examples of environmental best practice in chemical manufacturing. These exciting, but rather disparate, developments have encouraged the Royal Society of Chemistry to establish a Green Chemistry Network and a new journal and resource Green Chemistry. The Network aims to help promote and publicize the principles and practice of green chemistry, facilitate technology transfer, and assist in the development of new educational material that teaches students how to practice chemistry with an awareness of the environment. The journal carries news, views, and information about relevant developments, as well as publishing high-quality, original science articles and reviews on research aimed at a reduction in the environmental impact resulting from the use or manufacture of chemicals. Further information about the various green chemistry research, networking, educational, and publishing activities at York can be found by consulting the web site.

(C) 2000 IUPAC, Pure and Applied Chemistry 72, 1313-1319 


\section{ACKNOWLEDGMENTS}

The authors gratefully acknowledge the support of the EPSRC, the RAEng, and their industrial sponsors, as well as their colleagues in the York Green Chemistry Group.

\section{REFERENCES}

1. R. A. Sheldon. Chemistry \& Industry 1,12 (1997).

2. J. H. Clark, D. J. MacQuarrie. Chem. Comm. 853, (1998).

3. A. Corma. Chem. Rev. 95, 559 (1995).

4. A. Corma, H. Garcia. Catal. Today 38, 257 (1997), and refs therein.

5. J. S. Beck, J. C. Vartuli, W. J. Roth, M. E. Leonowicz, C. T. Kresge, K. D. Schmitt, C. T-W. Chu, D. H. Olsen, E. W. Sheppard, S. B. McCullen, J. B. Higgins, J. L. Schlenker. J. Am. Chem. Soc. 114, 10834 (1992).

6. T. Cseri, S. Bekassy, F. Figeuras, E. Cseke, L. C. Demenorval, R. Dunarte. Appl. Catal. A 132, 141 (1995).

7. A. Corma, V. Fornes, M. T. Navarro, J. Perez-Pariente. J. Catal. 148, 569 (1994).

8. E. Armengol, M. L. Canto, H. Garcia, M. T. Navarro. J. Chem. Soc. Chem. Comm. 519 (1995).

9. E. Armengol, A. Corma, H. Garcia, J. Primo. Appl. Cat. A. 126, 391, (1995).

10. E. Armengol, A. Corma, H. Garcia, J. Primo. Appl. Cat. A. 129, 411, (1997).

11. E. A. Gunnewegh, S. S. Gopie, H. Van Bekkum. J. Mol. Cat. A. 106, 151, (1996).

12. K. R. Kloestra and H. VanBekkum. J. Chem. Res. 1, 26 (1995).

13. M. J. Climent, A. Corma, S. Iborra, M. T. Navarro, J. Primo. J. Catal. 161, 786, (1996).

14. M. J. Climent, A. Corma, S. Iborra, S. Miquel, J. Primo, F. Ray. J. Catal. 183, 76 (1999).

15. P. T. Tanev and T. J. Pinnavaia. Science 267, 865 (1995).

16. J. P. Blitz and C. P. Little. Fundamentals and Applied Aspects of Chemically Modified Surfaces, Royal Society of Chemistry, Cambridge (1999).

17. L. A. Ciolino and J. G. Dorsey. J. Chromatr, A 675, 29 (1994).

18. W. M. VanRhijn, D. E. DeVos, B. F. Sels, W. D. Bossaert, P. A. Jacobs. Chem. Comm. 317 (1998).

19. W. D. Bossaert D. E. DeVos, W. M. VanRhijn, J. Bullen, P. J. Grobet, P. A. Jacobs. J. Catal. 182, 156 (1999).

20. G. A. Olah, P. S. Iyer, G. K. S. Prakash. Synthesis 513 (1986).

21. M. A. Harmer, W. E. Farmeth, Q. Sun. J. Am. Chem. Soc. 118, 7708 (1996).

22. Q. Sun, M. A. Harmer, W. E. Farneth. Chem. Comm. 1201 (1996).

23. M. A. Harmer, W. E. Farmeth, Q. Sun. Adv. Materials 10, 1255 (1998).

24. A. Heidekum, M. A. Harmer, W. F. Hoelderich. J. Catal. 176, 260 (1998).

25. M. A. Harmer, Q. Sun., M. J. Michalczyk, Z. Yang. Chem. Comm. 1803 (1997).

26. J. H. Clark, J. C. Ross, D. J. Macquarrie, S. J. Barlow, T. W. Bastock. Chem. Comm. 1203 (1997).

27. Y. Okamoto, T. Imanaka, S. Teranishi. Bull. Chem. Soc. Jpn. 47, 464 (1973).

28. J. H. Clark. Catalysis of Organic Reactions Using Supported Inorganic Reagents, $\mathrm{VCH}$, New York (1994).

29. K. Wilson, A. Renson, J. H. Clark. Catal. Lett. 61, 51 (1999).

30. D. Genske, K. Bornholdt, H. Lechert. Stud. Surf. Sci. Catal. 117, 44 (1998).

31. G. Gerstberger, C. Palm, R. Anwander. Chem. A - Eur. Jnl. 5, 997 (1995).

32. J. H. Clark, K. Martin, A. J. Teasdale, S. J. Barlow. J. Chem. Soc. Chem. Comm. 2037 (1995).

33. J. H. Clark, P. M. Price, K. Martin, D. J. Macquarrie, T. W. Bastock. J. Chem. Res. 430 (1997).

34. J. H. Clark, P. M. Price, K. Martin, D. J. Macquarrie, T. W. Bastock. UK Pat. Appl. (1996). 
35. B. M. Khadilkar and S. D. Borkar. Tetrahedron Lett. 38, 1641 (1997).

36. K. Wilson and J. H. Clark. Chem. Comm. 2135 (1998).

37. K. Wilson and J. H. Clark. UK Patent Application, GB9819456. 6 (1998).

38. B. A. Morrow and A. Devi. J. Chem. Soc. Faraday Trans. 68, 403 (1972).

39. M. Marczewwski, H. Marczewska, K. Witoslawski. Bull. Soc. Chim. Fr. 366 (1991).

40. K. Wilson, S. Pezin, J. H. Clark. In preparation.

41. M. Misono and T. Okuhara. Chem. Tech. 23 (1993).

42. I. V. Kozhevnikov. Chem. Rev. 98, 171 (1998).

43. Y. Izumi, K. Urabe, M. Onaka. Zeolite, Clay and Heteropoly Acid in Organic Reactions, Kodansha/ VCH, Tokyo, 99 (1992).

44. Y. Izumi. Catal. Today 33, 371 (1997).

45. I. V. Kozhevnikov and M. N. Timofeeva. J. Mol. Cat. A. 45, 179 (1992).

46. A. Gutierrez, S. L. Soled, J. A. Paes. U.S. Patent 5,334 775 (1994).

47. M. A. Schwegler, H. van Bekkum, N. deMunck. Appl. Catal. 74, 191 (1991).

48. M. J. Verhoet, P. Kooyman, J. A. Peters, H. VanBekkum. Micoporous \& Mesopourous Mat. 27, 365 (1999).

49. G. J. Meuzelaar, L. Maat, R. A. Sheldon, I. V. Kozhevnokov. Catal. Lett. 45, 249 (1997).

(C) 2000 IUPAC, Pure and Applied Chemistry 72, 1313-1319 